\title{
Ecologia da vegetação herbácea de bancadas lateríticas (cangas) em Corumbá, MS, Brasil
}

\author{
Adriana Takahasi ${ }^{1,3}$ e Sergio Tadeu Meirelles ${ }^{2}$
}

Recebido: 11.10.2013; aceito: 28.04.2014

\begin{abstract}
Ecology of herbaceous vegetation on bancadas lateríticas (cangas) in Corumbá, Mato Grosso do Sul State, Brazil). Bancadas lateríticas are ironstone outcrops that surround Morraria do Urucum and Morraria do Rabicho. This paper analyzes the floristic composition and structure of three ironstone outcrops, in quadrats of $1 \mathrm{~m}^{2}$, and reveals possible intrinsic patterns in site subsets by means of ordination analysis (detrended correspondence analysis - DCA) using cover data of plant species. A total of 66 vascular species belonging to 27 botany families were sampled. The richest families were Fabaceae (11 species) and Poaceae (9) while Selaginella sellowii and Sporobolus monandrus had the highest importance values. The ordination analysis suggested that herbaceous communities were influenced by the granulometry of the substrate, with species that occurred directly on hardened substrate and others that occurred on a thin soil layer. We emphasize the importance of the conservation of this flora, due both to its floristic insularity and to the little knowledge about the ecosystems that form the landscape mosaic of the Pantanal.
\end{abstract}

Keywords: ferricrete, ferruginous geosystem, ferruginous grasslands, phytosociology

RESUMO - (Ecologia da vegetação herbácea de bancadas lateríticas (cangas) em Corumbá, MS, Brasil). As bancadas lateríticas são carapaças ferruginosas que circundam as morrarias do Urucum e Rabicho. Este estudo verificou a composição florística e estrutura em três bancadas lateríticas, utilizando parcelas de $1 \mathrm{~m}^{2}$, e analisou a distribuição das espécies ao longo do gradiente ambiental através da análise de correspondência segmentada (DCA) para dados de cobertura. Amostraram-se 66 espécies vasculares pertencentes a 27 famílias. As famílias mais ricas foram Fabaceae (11 espécies) e Poaceae (9) enquanto Selaginella sellowii e Sporobolus monandrus apresentaram os maiores valores de importância. Os resultados da análise de ordenação sugerem que a vegetação herbácea é influenciada pela granulometria do substrato, com espécies que se estabelecem diretamente sobre o substrato endurecido e outras em locais com uma fina camada de sedimentos. Destaca-se, ainda, a importância da conservação desta flora, tanto pelo seu caráter insular quanto pela escassez de informações sobre estes ecossistemas que constituem o mosaico de paisagens do Pantanal.

Palavras-chave: Campo Ferruginoso, Ferricrete, Fitossociologia, Geossistema Ferruginoso

\section{Introdução}

Os afloramentos rochosos concentram-se em topos de morros e outras elevações distribuídos por todo o globo (Porembski et al. 1998), especialmente nas regiões tropicais (Barthlott et al. 1993a, Porembski et al. 1997, Porembski 2007). Nos neotrópicos distribuem-se pelo leste do Brasil, oeste da Venezuela e borda leste das Guianas; enquanto nos paleotrópicos ocorrem na África e Madagascar e, ainda, na Índia, Sri Lanka, China, Malásia e Austrália (Barthlott et al. 1993a).
As condições ambientais dos afloramentos rochosos, assaz adversas para uma comunidade de plantas, devem-se à grande amplitude térmica, às elevadas taxas de insolação, à inexistência ou exiguidade de solo e ao intenso déficit hídrico (Sarthou et al. 2009). Assim, os primeiros organismos a colonizar as rochas nuas são cianobactérias e liquens e, posteriormente, espécies pecilohídricas ou suculentas se estabelecem em pequenas depressões, cavidades ou fissuras da rocha (Hambler 1964, Meirelles et al. 1999). Graças à presença de primeiras plantas vasculares ali instaladas inicia-se a formação de solo

1. Universidade Federal de Mato Grosso do Sul, Laboratório de Botânica, câmpus do Pantanal, av. Rio Branco, 1270, 79304-020, Corumbá, MS, Brasil

2. Universidade de São Paulo, Instituto de Biociências, Departamento de Ecologia, Cidade Universitária, rua do Matão, travessa 14, 321, 05508-090 São Paulo, SP, Brasil

3. Autor para correspondência: adriana.takahasi@ufms.br 
que, por sua vez, permite que outras espécies vegetais - inclusive arbóreas - se estabeleçam. Assim, aquelas condições ambientais desfavoráveis são tornadas amenas nos agrupamentos de vegetação ou ilhas de solo (Conceição et al. 2007), propiciando uma complexificação crescente do ambiente com o decorrer do tempo. Evidentemente, tal processo está sujeito a ser perturbado em maior ou menor grau por influências diversas, desde a ação do homem a fenômenos naturais (Seine et al. 1998).

As plantas estabelecem-se em quatro tipos principais de habitats nos afloramentos rochosos: superfície rochosa, fissuras, depressões e agrupamentos (Seine et al. 1998) e, em função das peculiaridades de cada habitat, determinados grupos de plantas desenvolvem habilidades para colonizá-lo. Estes habitats podem ser subdivididos de acordo com as características do afloramento em estudo e dos objetivos da pesquisa, como por exemplo: vegetação criptogâmica, vegetação em canais de drenagem, vegetação efêmera durante a estação chuvosa, vegetação vascular litofítica, vegetação em fendas e fissuras, vegetação em poças sazonais, vegetação em fragmentos de rocha, vegetação em depressões preenchidas por solo, vegetação em agrupamentos ou ilhas de solo (Barthlott et al. 1993b, Porembski et al. 1994, Porembski et al. 1997, Porembski 2007).

No Brasil, as lateritas endurecidas (cangas) ocorrem principalmente no Planalto Central e na região Nordeste, enquanto na região amazônica sua distribuição se dá de forma mais ampla como plintita (laterita não endurecida) (Bigarella et al. 2007). Nestes locais, especificamente no Quadrilátero Ferrífero (MG), na Serra de Carajás (PA), na região de Caetité (BA) e na Morraria do Urucum (MS) (Carmo et al. 2012), encontram-se as maiores reservas de minério de ferro do país, cuja exploração econômica, sem o devido planejamento ambiental, tem sido a principal ameaça à flora destes habitats (Jacobi et al. 2007). Embora a legislação ambiental brasileira determine a recuperação de áreas degradadas por mineração, o potencial da vegetação sobre cangas, para este propósito, ainda é pouco explorado (Jacobi et al. 2008).

A vegetação que se estabelece sobre estes substratos ferruginosos endurecidos foi classificada por Rizzini (1997) como campo ferruginoso, englobando dois subtipos: campo de canga couraçada (couraça laterítica) e campo de canga nodular (couraça fragmentada). Várias terminologias têm sido empregadas para se referir à formação vegetal associada às cangas, como vegetação de canga (Secco \& Mesquita 1983, Silva 1991, Carmo \& Jacobi 2013), campo rupestre ferruginoso (Secco \& Lobo 1988, Cleef \& Silva 1994, Silva et al. 1996, Jacobi et al. 2007, Mourão \& Stehmann 2007, Viana \& Lombardi 2007, Jacobi \& Carmo 2008, Jacobi et al. 2008, Vincent \& Meguro 2008, Messias et al. 2012, Messias et al. 2013), vegetação xerofítica de savanas metalófitas (Rayol 2006) e vegetação de bancada laterítica (Silva \& Tozzi 2008, Takahasi 2010). A imprecisão e variedade na nomenclatura empregada para referir-se à vegetação sobre os substratos ferruginosos endurecidos refletem a controvérsia da aplicação do conceito de laterita (ver Augustin et al. 2013 para maiores detalhes) e, obviamente, à heterogeneidade geológica e geomorfológica das cangas. Recentemente, alguns autores têm proposto uma classificação atualizada da flora associada às cangas do Quadrilátero Ferrífero considerando-as componentes dos geossistemas ferruginosos (Carmo \& Jacobi 2013).

A elevada concentração de metais pesados nestes ambientes influencia a composição florística reforçando o grau de insularidade destes locais (já que soa como cafonia) tendo em vista que a flora das couraças, e dos afloramentos rochosos de modo geral, tende a ser distinta daquela do entorno (Porto \& Silva 1989, Silva 1992, Jacobi et al. 2007, Vincent $\&$ Meguro 2008). Tais plantas podem ser indiferentes ao ambiente rico em metais pesados ou, ainda, pouco afetadas pela entrada de uma concentração mais elevada (Ginocchio \& Baker 2004). Sugere-se que as condições peculiares no metabolismo destas plantas explicam a ocorrência de endemismos nos afloramentos ferríferos (Porto \& Silva 1989, Silva 1992) e em locais com solos metalíferos (Ginocchio \& Baker 2004), onde algumas espécies de plantas poderiam, inclusive, ser utilizadas como indicadoras de jazidas de minerais (Baker \& Brooks 1988, Silva 1992, Ginocchio \& Baker 2004).

Estudos recentes têm demonstrado a heterogeneidade e diversidade das comunidades vegetais sobre substratos ferruginosos endurecidos no Quadrilátero Ferrífero no Estado de Minas Gerais (Jacobi et al. 2007, Mourão \& Stehmann 2007, Viana \& Lombardi 2007, Jacobi et al. 2008, Vincent \& Meguro 2008, Messias et al. 2012, Carmo \& Jacobi 2013); na Serra do Espinhaço (Messias et al. 2013) e na Serra de Carajás no Pará (Rayol 2006), com o 
registro de alguns endemismos (Viana \& Lombardi 2007, Jacobi \& Carmo 2008). Na região de Corumbá, estas comunidades vegetais têm sido pouco estudadas (Takahasi 2010) e, embora as atividades mineradoras não afetem diretamente estas comunidades, elas apresentam-se pouco preservadas tanto por atividades extrativistas (especialmente o comércio de orquídeas e cactos), quanto por pastoreio pelo gado, destruição pelo fogo ou construção de casas pelos proprietários destas áreas.

As cangas das Morrarias do Urucum, do Rabicho e Amolar (MS)são denominadas bancadas lateríticas e localizam-se nas áreas de drenagem (ca. de 100 m de altitude) no sopé destes morros, possuem pequena declividade e são formados por material laterítico endurecido de natureza ferrífera (Cardoso et al. 2000), com pouca aptidão para usos agrícolas ou pastoris (Pott et al. 2000).

Estes substratos ferruginosos endurecidos podem ser considerados ecossistemas similares àqueles em afloramentos rochosos, onde as plantas vasculares se estabelecem diretamente sobre o substrato endurecido ou entre fragmentos de rochas ou na forma de agrupamentos ("mats") referidos em diversos estudos como ilhas de solo (Burbanck \& Platt 1964, Shure \& Ragsdale 1977, Phillips 1981, Meirelles et al. 1999, Conceição et al. 2007, Ribeiro et al. 2007, Sarthou et al. 2009, Takahasi 2010).

O presente estudo apresenta dados quantitativos sobre a comunidade herbácea das bancadas lateríticas de Corumbá e Ladário, no Estado de Mato Grosso do Sul e, desta forma, busca contribuir para ampliar o conhecimento sobre a flora destes ambientes ferruginosos no Brasil. Este trabalho teve por objetivos: verificar a composição florística e a estrutura das bancadas lateríticas e revelar padrões de distribuição das espécies ao longo gradiente ambiental em três localidades.

\section{Material e métodos}

Área de estudo - Analisou-se a vegetação herbácea de três bancadas lateríticas nos municípios de Corumbá (fazenda Monjolinho $19^{\circ} 16^{\prime} \mathrm{S}, 57^{\circ} 31^{\prime} \mathrm{W}$, 65-150 m.s.m.) e Ladário (fazenda Banda Alta $19^{\circ} 08^{\prime} \mathrm{S}, 57^{\circ} 34^{\prime} \mathrm{W}, 85$ m.s.m. - e fazenda São Sebastião do Carandá - $19^{\circ} 06^{\prime} \mathrm{S}, 57^{\circ} 31^{\prime} \mathrm{W}, 90$ m.s.m.), Estado do Mato Grosso de Sul. As duas primeiras localidades correspondem a um campo sobre canga couraçada, enquanto a última área é um campo sobre canga nodular.
O clima de Corumbá pode ser classificado pelo sistema de Köppen como Awa correspondendo a um clima tropical megatérmico (a temperatura do mês mais frio é superior a $18^{\circ} \mathrm{C}$ ) com inverno seco e chuvas no verão (Soriano 2000). A precipitação média anual de Corumbá é de $1.070 \mathrm{~mm}$ anuais e a temperatura média anual é de $25,1{ }^{\circ} \mathrm{C}$, oscilando entre 21,4 e $27,7^{\circ} \mathrm{C}$ (Soriano 2000).

Estudos florísticos - A área total de cada bancada laterítica foi medida em seu maior comprimento e largura para a elaboração de uma grade de $5 \mathrm{~m}$ utilizada para o sorteio dos locais para instalação de parcelas. A verificação da composição e da estrutura do estrato herbáceo foram avaliadas em 199 parcelas de $1 \times 1 \mathrm{~m}$, sendo alocadas 81 parcelas na fazenda Banda Alta, 60 na fazenda São Sebastião do Carandá e 58 na fazenda Monjolinho. Se o ponto de amostragem sorteado foi um local sem vegetação vascular ou uma ilha de solo ele foi descartado. Assim, o número total de parcelas em cada local variou em função da área total de substrato ferruginoso endurecido e da quantidade de ilhas de solo presentes.

Foram identificados dois habitats na fazenda Banda Alta e por isso a amostragem foi estratificada. No primeiro ambiente, denominado habitat 'seco', a superfície da canga é relativamente lisa e homogênea, com alguns canais de drenagem e pequenas depressões, e constitui a maior porção da canga. O segundo habitat, denominado 'sazonalmente inundado', próximo ao Córrego Band'Alta, caracteriza-se por uma superfície laterítica bastante irregular, com muitas macrocavidades que permitem a deposição de sedimentos e matéria orgânica oriundos das partes mais elevadas. Dada sua posição no relevo, este local recebe e retém a água das chuvas vários dias após a interrupção das mesmas. Desta forma, várias espécies de plantas típicas de ambientes sazonalmente inundáveis ocorrem neste local. Para verificar se a cobertura e a composição de espécies destes dois habitats eram significantemente diferentes foi empregada uma análise de variância entre o tipo de habitat e os escores dos dois primeiros eixos da DCA, elaborada a partir da matriz de dados de cobertura padronizados por valor máximo para as espécies com ocorrência em mais de três unidades amostrais (ver tópico "análise de gradientes" para maiores detalhes).

Foram registradas as espécies vasculares presentes em cada unidade amostral e estimou-se sua cobertura vegetal, definida como a projeção vertical 
da área ocupada por ramos ou copa dos indivíduos na superfície do solo, expressa como porcentagem (Mueller-Dombois \& Ellenberg 1974).

No interior de cada unidade amostral foram estimadas a cobertura de substrato ferruginoso endurecido, de sedimentos e de presença de fragmentos de minério de ferro e manganês endurecidos, maiores do que $2 \mathrm{~cm}$, para análise do gradiente ambiental (DCA), mas estes dados não foram computados nas análises quantitativas.

Os dados quantitativos foram avaliados no período de fevereiro de 2008 a março de 2009. Foram calculados os seguintes parâmetros fitossociológicos: frequência e dominância (cobertura), absolutas e relativas, e o valor de importância das espécies (Mueller-Dombois \& Ellenberg 1974). Os valores de frequência foram obtidos a partir do número de unidades amostrais em que a espécie estava presente e os valores de dominância foram obtidos a partir do grau de cobertura ocupada pela espécie em cada parcela. A densidade de espécies não foi determinada devido à dificuldade de individualização de plantas herbáceas que se propagam vegetativamente por órgãos subterrâneos. Assim, o índice do valor de importância (Mueller-Dombois \& Ellenberg 1974) foi modificado considerando-se a soma da frequência e dominância relativas, procedimento adotado em outros estudos fitossociológicos, como por exemplo, o trabalho de Caiafa \& Silva (2007) em campos de altitude.

A diversidade específica da comunidade herbácea sobre canga foi expressa pelo índice de diversidade de Shannon-Wiener (H') na base logarítmica natural com uma modificação utilizando-se, ao invés de número de indivíduos, os valores de cobertura amostrados. O índice de equabilidade de Pielou (J) foi utilizado para estimativa da uniformidade das comunidades (Krebs 1972).

O material testemunho foi depositado no Herbário COR (UFMS, câmpus do Pantanal, Corumbá, MS) com duplicatas enviadas aos especialistas para identificação. A circunscrição de famílias baseou-se em APG III (2009), e o nome dos autores de espécies foi verificado em Brummitt \& Powell (1992) e em bases de dados contidas nas páginas eletrônicas: Lista de espécies da flora do Brasil (2014), The plant list (2014) e Tropicos.org (2014).

Análise de gradientes - As relações entre a cobertura vegetal e a composição de espécies das parcelas dos três locais foram exploradas pela análise de correspondência segmentada (DCA), empregando-se o algoritmo de Hill com a correção de tendência ("detrending"), empregando-se a partição em 26 segmentos para recálculo com 100 iterações (Legendre \& Legendre 1998). Para tanto, a matriz contendo valores de cobertura de espécies por parcela foi reduzida, considerando-se apenas aquelas espécies com três ocorrências ou mais, a fim de eliminar interferências das espécies raras nas análises. Em seguida, a matriz foi padronizada por valor máximo no espaço das variáveis. Como a composição florística das três localidades foi bastante distinta, optou-se por fazer a análise de gradiente em cada local, a fim de minimizar a interferência desta variável. As análises de ordenação (DCA) foram realizadas com auxílio do programa MVSP for Windows versão 3.1 (Kovach Computing Services).

\section{Resultados}

Fitossociologia e estrutura - Foram registradas 66 espécies pertencentes a 27 famílias botânicas, sendo cinco espécies de pteridófitas pertencentes a três famílias, numa área total amostrada de $199 \mathrm{~m}^{2}$ (tabela 1).

A família com maior número de espécies foi Fabaceae (11 espécies), seguida por Poaceae (9), além de Cyperaceae, Malvaceae e Portulacaceae (5 espécies cada).

Do total amostrado foram contabilizados $35,4 \mathrm{~m}^{2}$ de substrato ferruginoso endurecido (sem cobertura vegetal), seguidos por 11,2 $\mathrm{m}^{2}$ de sedimentos (sem plantas), além de $2,3 \mathrm{~m}^{2}$ de fragmentos de minério de ferro e manganês endurecidos, ou seja, cerca de $25 \%$ da área total amostrada não apresentou cobertura vegetal. A fazenda Banda Alta teve a maior proporção de área amostrada sem plantas vasculares $\left(36 \mathrm{~m}^{2}\right)$, seguida pela fazenda Monjolinho $\left(7 \mathrm{~m}^{2}\right)$ e pela fazenda São Sebastião do Carandá $\left(6 \mathrm{~m}^{2}\right)$.

As dez espécies com maiores valores de frequência relativa, totalizando $58,6 \%$, foram: Selaginella sellowii (11,6\%), Mimosa nuda (6,4\%), Sporobolus monandrus (6,2\%), Tripogon spicatus (6\%), Mimosa xanthocentra (5,4\%), Staelia sp. $(5,1 \%)$, Waltheria operculata (5\%), Jacquemontia evolvuloides (5\%), Aeschynomene histrix $(4,4 \%) \mathrm{e}$ Portulaca mucronulata (3,3\%) (tabela 1; figura 1).

As dez espécies com maiores valores de dominância relativa perfizeram $86,3 \%$ do total: Selaginella sellowii (32,9\%), Sporobolus monandrus (16,4\%), Tripogon spicatus (6,9\%), Mesosetum chasae (5,9\%), Mimosa nuda (5,5\%), Mimosa xanthocentra 
Tabela 1. Parâmetros fitossociológicos para as espécies vasculares amostradas nas bancadas lateríticas em Corumbá e Ladário, MS, Brasil. ABREV.: abreviatura do nome científico; UA: número de unidades amostrais em que ocorre a espécie; FA: frequência absoluta (\%); FR: frequência relativa (\%); DoA: dominância absoluta $\left(\mathrm{m}^{2}\right)$; DoR: dominância relativa $(\%)$; VI: valor de importância (\%); N.col.: número de coletor de Adriana Takahasi.

Table 1. Quantitative descriptors for vascular plants sampled in ironstone outcrops of Corumbá and Ladário, Mato Grosso Sul State, Brazil. ABREV.: scientific name; UA: number of quadrats where species occurred; FA: absolute frequency (\%); FR: relative frequency (\%); DoA: absolute dominance (m2 ); DoR: relative dominance (\%); VI: importance value index (\%); N.col.: voucher of Adriana Takahasi.

\begin{tabular}{|c|c|c|c|c|c|c|c|c|}
\hline Família/Espécie & ABREV. & UA & FA & FR & DoA & DoR & VI & N.col. \\
\hline \multicolumn{9}{|l|}{ AMARANTHACEAE } \\
\hline Gomphrena centrota E. Holzh. & GOM & 20 & 10,05030 & 1,48148 & 0,0071 & 0,71302 & 1,0973 & 1040 \\
\hline \multicolumn{9}{|l|}{ AMARYLLIDACEAE } \\
\hline Zephyranthes cearensis (Herb.)Baker & ZEP & 7 & 3,51759 & 0,51852 & 0,0015 & 0,14994 & 0,3342 & 947 \\
\hline \multicolumn{9}{|l|}{ ASTERACEAE } \\
\hline Calea elongata (Gardner) Baker & CAL & 9 & 4,52261 & 0,66667 & 0,0029 & 0,28654 & 0,4766 & 1039 \\
\hline Lepidaploa salzmannii (DC.) H.Rob. & LEP & 11 & 5,52764 & 0,81481 & 0,001 & 0,10329 & 0,4591 & 714 \\
\hline \multicolumn{9}{|l|}{ BIXACEAE } \\
\hline Cochlospermum regium (Schrank) Pilg. & & 1 & 0,50251 & 0,07407 & 0,0001 & 0,01333 & 0,0437 & 705 \\
\hline \multicolumn{9}{|l|}{ CACTACEAE } \\
\hline $\begin{array}{l}\text { Discocactus ferricola Buining \& } \\
\text { Brederoo }\end{array}$ & DIS & 23 & 11,55780 & 1,7037 & 0,0115 & 1,1495 & 1,4266 & 845 \\
\hline \multicolumn{9}{|l|}{ CARYOPHYLLACEAE } \\
\hline Polycarpaea corymbosa (L.) Lam. & $\mathrm{PCO}$ & 15 & 7,53769 & 1,11111 & 0,0019 & 0,18992 & 0,6505 & 973 \\
\hline \multicolumn{9}{|l|}{ CLEOMACEAE } \\
\hline Cleome eosina J.F. Macbr. & CLE & 3 & 1,50754 & 0,22222 & 0,0007 & 0,0733 & 0,1478 & 829 \\
\hline Cleome guianensis Aubl. & CLG & 23 & 11,55780 & 1,7037 & 0,0036 & 0,35651 & 1,0301 & 780 \\
\hline \multicolumn{9}{|l|}{ CONVOLVULACEAE } \\
\hline Evolvulus alopecuroides Mart. & & 14 & 7,03518 & 1,03704 & 0,0018 & 0,17659 & 0,6068 & 1383 \\
\hline Evolvulus filipes Mart. & EVF & 5 & 2,51256 & 0,37037 & 0,0002 & 0,02332 & 0,1968 & 1203 \\
\hline Jacquemontia evolvuloides Meisn. & JAE & 68 & 34,17090 & 5,03704 & 0,0342 & 3,42185 & 4,2294 & 1125 \\
\hline Jacquemontia fruticulosa Hallier f. & & 1 & 0,50251 & 0,07407 & $3 \mathrm{E}-05$ & 0,00333 & 0,0387 & 940 \\
\hline \multicolumn{9}{|l|}{ CYPERACEAE } \\
\hline Cyperus aggregatus (Willd.) Endl. & & 37 & 18,59300 & 2,74074 & 0,0067 & 0,67437 & 1,7076 & 1363 \\
\hline Cyperus cornelii-ostenii Kük. & CYC & 5 & 2,51256 & 0,37037 & 0,001 & 0,09662 & 0,2335 & 1343 \\
\hline Cyperus uncinulatus Schrad. ex Nees & CYU & 41 & 20,60300 & 3,03704 & 0,0392 & 3,92497 & 3,481 & 1129 \\
\hline Fimbristylis sp. & FIM & 9 & 4,52261 & 0,66667 & 0,0013 & 0,12994 & 0,3983 & 1338 \\
\hline Kyllinga odorata Vahl & KYL & 4 & 2,01005 & 0,2963 & 0,0009 & 0,08663 & 0,1915 & 1268 \\
\hline \multicolumn{9}{|l|}{ EUPHORBIACEAE } \\
\hline Croton pedicellaltus Kunth & CRP & 7 & 3,51759 & 0,51852 & 0,0011 & 0,10662 & 0,3126 & 943 \\
\hline \multicolumn{9}{|l|}{ IRIDACEAE } \\
\hline Cipura formosa Ravenna & CIP & 4 & 2,01005 & 0,2963 & 0,0003 & 0,02666 & 0,1615 & 865 \\
\hline \multicolumn{9}{|l|}{ LAMIACEAE } \\
\hline Hyptis brevipes Poit. & & 1 & 0,50251 & 0,07407 & 0,0001 & 0,00666 & 0,0404 & 1012 \\
\hline
\end{tabular}


Tabela 1 (continuação)

\begin{tabular}{|c|c|c|c|c|c|c|c|c|}
\hline Família/Espécie & ABREV. & UA & FA & FR & DoA & DoR & VI & N.col. \\
\hline \multicolumn{9}{|l|}{$\begin{array}{l}\text { LEGUMINOSAE } \\
\end{array}$} \\
\hline Aeschynomene histrix Poir. & AES & 60 & 30,1508 & 4,44444 & 0,0156 & 1,55932 & 3,0019 & 925 \\
\hline Alysicarpus vaginalis (L.) DC. & ALY & 8 & 4,0201 & 0,59259 & 0,0007 & 0,0733 & 0,3329 & 1019 \\
\hline Bauhinia pentandra (Bong.) Steud. & & 1 & 0,50251 & 0,07407 & $7 \mathrm{E}-05$ & 0,00666 & 0,0404 & 961 \\
\hline Camptosema ellipticum (Desv.) Burkart & & 1 & 0,50251 & 0,07407 & $7 \mathrm{E}-05$ & 0,00666 & 0,0404 & 1041 \\
\hline Centrosema pascuorum Mart. ex Benth. & & 2 & 1,00503 & 0,14815 & $1 \mathrm{E}-04$ & 0,01 & 0,0791 & 1373 \\
\hline Chamaecrista flexuosa (L.) Greene & $\mathrm{CHF}$ & 11 & 5,52764 & 0,81481 & 0,0021 & 0,20991 & 0,5124 & 1107 \\
\hline Chamaecrista serpens (L.) Greene & CHS & 4 & 2,01005 & 0,2963 & 0,0004 & 0,04331 & 0,1698 & 1006 \\
\hline Mimosa nuda Benth. & $\mathrm{MNU}$ & 86 & 43,2161 & 6,37037 & 0,0548 & 5,48762 & 5,929 & 1050 \\
\hline Mimosa xanthocentra Mart. & MXA & 73 & 36,6834 & 5,40741 & 0,0535 & 5,35435 & 5,3809 & 958 \\
\hline $\begin{array}{l}\text { Stylosanthes acuminata M. B. Ferreira } \\
\text { \& S. Costa }\end{array}$ & STY & 31 & 15,5779 & 2,2963 & 0,0162 & 1,62263 & 1,9595 & 956 \\
\hline Zornia reticulata $\mathrm{Sm}$. & ZOR & 32 & 16,0804 & 2,37037 & 0,008 & 0,79632 & 1,5833 & 1134 \\
\hline \multicolumn{9}{|l|}{ LYTHRACEAE } \\
\hline Cuphea cf. micrantha Kunth & & 3 & 1,50754 & 0,22222 & 0,0003 & 0,02666 & 0,1244 & 785 \\
\hline \multicolumn{9}{|l|}{ MALPIGHIACEAE } \\
\hline $\begin{array}{l}\text { Aspicarpa pulchella O'Donell \& } \\
\text { Lourteig }\end{array}$ & & 2 & 1,00503 & 0,14815 & 0,0005 & 0,05331 & 0,1007 & 769 \\
\hline Janusia guaranitica (A. St.-Hil.) A. Juss. & & 1 & 0,50251 & 0,07407 & 0,0001 & 0,00333 & 0,0387 & 819 \\
\hline \multicolumn{9}{|l|}{ MALVACEAE } \\
\hline Ayenia tomentosa $\mathrm{L}$. & AYE & 6 & 3,01508 & 0,44444 & 0,0007 & 0,0733 & 0,2589 & 1081 \\
\hline Sida cordifolia $\mathrm{L}$. & & 1 & 0,50251 & 0,07407 & 0,0001 & 0,00333 & 0,0387 & 1279 \\
\hline Sida glomerata Cav. & & 1 & 0,50251 & 0,07407 & 0,0001 & 0,00333 & 0,0387 & 1281 \\
\hline Waltheria operculata Rose & WAL & 68 & 34,1709 & 5,03704 & 0,01 & 1,0029 & 3,02 & 771 \\
\hline Waltheria sp. & WAT & 4 & 2,01005 & 0,2963 & 0,0004 & 0,03665 & 0,1665 & 942 \\
\hline \multicolumn{9}{|l|}{ MOLLUGINACEAE } \\
\hline Mollugo verticillata $\mathrm{L}$. & MOL & 23 & 11,5578 & 1,7037 & 0,0035 & 0,35318 & 1,0284 & 839 \\
\hline \multicolumn{9}{|l|}{ OPHIOGLOSSACEAE } \\
\hline Ophioglossum sp. & OPH & 3 & . 1,50754 & 0,22222 & 0,0004 & 0,03998 & 0,1311 & 1262 \\
\hline \multicolumn{9}{|l|}{ OXALIDACEAE } \\
\hline Oxalis frutescens $\mathrm{L}$. & OX1 & 4 & 2,01005 & 0,2963 & 0,0002 & 0,02332 & 0,1598 & 1106 \\
\hline \multicolumn{9}{|l|}{ PHYTOLACCACEAE } \\
\hline Microtea scabrida Urb. & MSC & 15 & 7,53769 & 1,11111 & 0,0011 & 0,11328 & 0,6122 & 910 \\
\hline \multicolumn{9}{|l|}{ POACEAE } \\
\hline Melinis repens (Willd.) Zizka & & 7 & 3,51759 & 0,51852 & 0,0021 & 0,21324 & 0,3659 & 747 \\
\hline Mesosetum chaseae Luces & MÊS & 42 & 21,1055 & 3,11111 & 0,0593 & 5,9341 & 4,5226 & 1204 \\
\hline Microchloa indica (L.f.) P. Beauv. & MIND & 30 & 15,0754 & 2,22222 & 0,0274 & 2,73881 & 2,4805 & 1172 \\
\hline Oplismenus hirtellus (L.) P. Beauv. & OPL & 5 & 2,51256 & 0,37037 & 0,0016 & 0,1566 & 0,2635 & 1274 \\
\hline Panicum exiguum $\mathrm{Mez}$ & PAE & 5 & 2,51256 & 0,37037 & 0,005 & 0,49645 & 0,4334 & 1150 \\
\hline
\end{tabular}


Tabela 1 (continuação)

\begin{tabular}{|c|c|c|c|c|c|c|c|c|}
\hline Família/Espécie & ABREV. & UA & FA & FR & DoA & DoR & VI & N.col. \\
\hline Paspalum malacophyllum Trin. & PMA & 6 & 3,01508 & 0,44444 & 0,0043 & 0,42648 & 0,4355 & 1273 \\
\hline $\begin{array}{l}\text { Sporobolus monandrus Roseng., } \\
\text { B.R.Arril. \& Izag. }\end{array}$ & SPO & 84 & 42,2111 & 6,22222 & 0,1637 & 16,3762 & 11,299 & 1145 \\
\hline Tripogon spicatus (Nees) Ekman & TRI & 81 & 40,7035 & 6 & 0,0685 & 6,85703 & 6,4285 & 1131 \\
\hline Urochloa adspersa (Trin.)R.D. Webster & & 2 & 1,00503 & 0,14815 & 0,0024 & 0,23656 & 0,1924 & 1277 \\
\hline \multicolumn{9}{|l|}{ PORTULACACEAE } \\
\hline Portulaca halimoides L. & & 2 & 1,00503 & 0,14815 & 0,0002 & 0,01999 & 0,0841 & 1340 \\
\hline Portulaca mucronata Link & & 1 & 0,50251 & 0,07407 & 0,0001 & 0,00666 & 0,0404 & 882 \\
\hline Portulaca mucronulata D.Legrand & PO1 & 45 & 22,6131 & 3,33333 & 0,0055 & 0,54976 & 1,9415 & 970 \\
\hline Portulaca pilosa $\mathrm{L}$. & PO5 & 8 & 4,0201 & 0,59259 & 0,0006 & 0,05997 & 0,3263 & 901 \\
\hline Portulaca umbraticola Kunth & & 1 & 0,50251 & 0,07407 & 0,0001 & 0,00333 & 0,0387 & 1367 \\
\hline \multicolumn{9}{|l|}{ RUBIACEAE } \\
\hline Borreria verticillata (L.)G.Mey. & BOR & 3 & 1,50754 & 0,22222 & 0,0001 & 0,01333 & 0,1178 & 1138 \\
\hline Staelia sp. & STA & 69 & 34,6734 & 5,11111 & 0,0325 & 3,25526 & 4,1832 & 874 \\
\hline \multicolumn{9}{|l|}{ SCHIZAEACEAE } \\
\hline Anemia tomentosa (Savigny) Sw. & ANE & 10 & 5,02513 & 0,74074 & 0,0051 & 0,51311 & 0,6269 & 858 \\
\hline \multicolumn{9}{|l|}{ SELAGINELLACEAE } \\
\hline Selaginella convoluta (Arn.) Spring & SEC & 15 & 7,53769 & 1,11111 & 0,002 & 0,19991 & 0,6555 & 1479 \\
\hline $\begin{array}{l}\text { Selaginella marginata (Humb. \& Bonpl. } \\
\text { ex Willd.) Spring }\end{array}$ & & 2 & 1,00503 & 0,14815 & 0,0007 & 0,06664 & 0,1074 & 750 \\
\hline Selaginella sellowii Hieron. & SEL & 157 & 78,8945 & 11,6296 & 0,329 & 32,9211 & 22,275 & 1311 \\
\hline Turnera pumilea $\mathrm{L}$. & TU2 & 10 & 5,02513 & 0,74074 & 0,0007 & 0,06664 & 0,4037 & 820 \\
\hline \multicolumn{9}{|l|}{ VERBENACEAE } \\
\hline $\begin{array}{l}\text { Stachytarpheta matogrossensis } \\
\text { Moldenke }\end{array}$ & STM & 17 & 8,54271 & 1,25926 & 0,0027 & 0,27321 & 0,7662 & 765 \\
\hline
\end{tabular}

(5,4\%), Cyperus uncinulatus (3,9\%), Jacquemontia evolvuloides (3,4\%), Staelia sp. (3,3\%) e Microchloa indica (2,7\%) (tabela 1). Duas espécies, Selaginella sellowii e Sporobolus monandrus, representaram cerca de $50 \%$ dos valores de dominância relativa.

As dez espécies com maiores valores de importância perfizeram um total de 30,2\%: Selaginella sellowii $(22,3 \%)$, Sporobolus monandrus $(11,3 \%)$, Tripogon spicatus (6,4\%), Mimosa nuda (5,9\%), Mimosa xanthocentra (5,4\%), Mesosetum chasae (4,5\%), Jacquemontia evolvuloides (4,2\%), Staelia sp. (4,2\%), Cyperus uncinulatus (3,5\%), Waltheria operculata (3\%) (tabela 1).

$\mathrm{O}$ valor do índice de diversidade de ShannonWiener (H') estimado para a vegetação herbácea foi de 2,4934 e o índice de equabilidade (J) foi igual a 0,5951.

Análise de gradiente da fazenda Banda Alta - Os autovalores dos dois primeiros eixos resultantes da análise de DCA da matriz de dados de cobertura de 32 espécies e dois atributos abióticos (substrato ferruginoso endurecido e sedimento) das 81 parcelas amostradas na fazenda Banda Alta foram 0,734 e 0,419, respectivamente, representando $18,1 \%$ da variação total da matriz, com $11,5 \%$ e $6,6 \%$, para o primeiro e segundo eixos, respectivamente. A interpolação entre os eixos 1 e 2 da DCA revelou um gradiente ambiental com as espécies amostradas nas parcelas do "habitat seco", no lado esquerdo do eixo 1, e as espécies das parcelas alocadas no "habitat sazonalmente inundado", no lado direito do eixo (figura 2.a). As espécies Discocactus ferricola, Gomphrena centrota, Polycarpaea corymbosa, Portulaca mucronulata e Selaginella sellowii tendem a apresentar maiores valores de cobertura em parcelas com maior proporção de substrato ferruginoso endurecido (figura 2.b). As espécies com reprodução por estruturas subterrâneas 
como, por exemplo, Cipura formosa e Zephyranthes cearensis e, outras, como as adaptadas a ambientes mais úmidos, Cyperus uncinullatus, Fimbristylis sp., Kyllinga odorata e Selaginella convoluta, tiveram maiores valores de cobertura em parcelas com maior proporção de solo coluvial (figura 2.b).

A análise de variância entre os escores dos dois primeiros eixos resultantes da DCA e o tipo de habitat (seco ou sazonalmente inundado) da fazenda

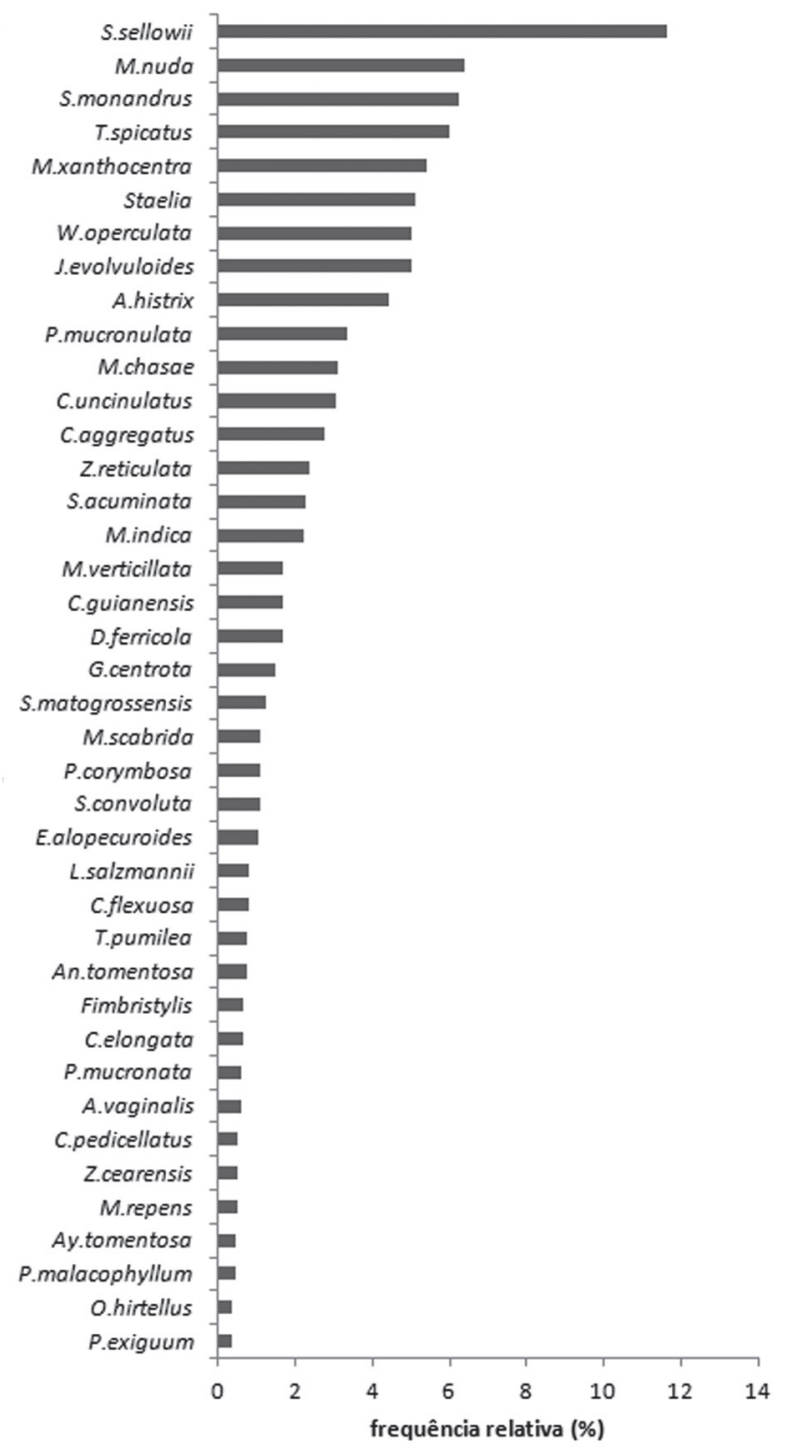

Figura 1. Valores de frequência relativa para as primeiras quarenta espécies, em porcentagem, da vegetação do estrato herbáceo de bancadas lateríticas amostrada em Corumbá e Ladário, MS, Brasil. Para o nome completo das espécies consultar a Tabela 1.

Figure 1. Relative frequency values for the first forty plant species of herbaceous vegetation from ironstone outcrops recorded in Corumbá and Ladário, Mato Grosso do Sul, Brazil. See table 1 for species names.
Banda Alta mostrou que a estrutura e composição florística destes dois habitats foram significativamente diferentes $(F=28,734$, g.l. $=80, p<0,001)$.

Análise de gradiente da fazenda São Sebastião do Carandá - Os autovalores dos dois primeiros eixos resultantes da análise de DCA da matriz de dados de cobertura de 24 espécies e dois atributos abióticos (substrato ferruginoso endurecido e sedimento) das 60 parcelas amostradas na fazenda São Sebastião do Carandá foram 0,672 e 0,381, respectivamente, representando $23,6 \%$ da variação total da matriz, com $15,1 \%$ e $8,5 \%$, respectivamente.

A interpolação entre os eixos 1 e 2 da DCA revelou que o substrato foi o principal fator responsável pela distribuição das espécies ao longo do eixo 1 , com as parcelas com espécies que se estabelecem diretamente sobre o substrato ferruginoso endurecido, Portulaca mucronulata e Selaginella sellowii, agrupadas no lado direito do eixo (figura 3.a). As demais parcelas com espécies associadas a um solo mais profundo distribuíram-se no lado esquerdo do eixo 1, em um gradiente em relação ao eixo 2 (figura 3.b), como Fimbristylis sp., Mimosa xanthocentra, Evolvulus alopecuroides, Jacquemontia evolvuloides, Staelia sp., Aeschynomene histrix, Stachytarpheta matogrossensis, Zornia reticulata, Mimosa nuda e Microtea scabrida.

Análise de gradiente da fazenda Monjolinho - Os autovalores dos dois primeiros eixos resultantes da análise de DCA da matriz de dados de cobertura de 29 espécies e três atributos abióticos (substrato ferruginoso endurecido, sedimentos e fragmentos de minério de ferro e manganês endurecidos) das 58 parcelas amostradas na fazenda Monjolinho foram 0,517 e 0,359 , respectivamente, representando $20,5 \%$ da variação total da matriz com $12,1 \%$ e $8,4 \%$ para os eixos 1 e 2, respectivamente (figura 4.a).

As análises de ordenação para a fazenda Monjolinho sugerem que a presença de sedimentos sobre a canga influencia a distribuição das espécies ao longo do gradiente ambiental (figura 4.b), onde as espécies Polycarpaea corymbosa e Cyperus cornelli-ostenii estiveram associadas a parcelas com maior proporção de substrato ferruginoso endurecido, enquanto Discocactus ferricola, Portulaca mucronulata e Selaginella sellowii estiveram relacionadas a parcelas com maiores proporções de sedimento e fragmentos de minério de ferro e manganês endurecidos. As parcelas com maior proporção de sedimentos no interior das 
parcelas estiveram associadas à presença das espécies Selaginella sellowii, Portulaca mucronulata, Tripogon spicatus e Microchloa indica (figura 4.b). Estas duas últimas espécies sempre ocorrem em locais com uma camada fina de substrato.

\section{Discussão}

As famílias botânicas com maior riqueza de espécies encontradas (Fabaceae e Poaceae) possuem uma ampla distribuição em diferentes formações vegetais tropicais, sendo Poaceae uma das famílias com maior número de espécies encontradas nas cangas de Carajás (Secco \& Mesquita 1983, Silva 1991) e do Quadrilátero Ferrífero (Jacobi et al. 2007, Viana \& Lombardi 2007, Jacobi et al. 2008, Messias et al. 2012, Carmo \& Jacobi 2013).

Destaca-se a ausência de espécies de Velloziaceae nas cangas de Corumbá, uma família botânica característica dos campos ferruginosos do Estado de Minas Gerais (Rizzini 1997, Jacobi et al. 2007, Mourão \& Stehmann 2007, Jacobi \& Carmo 2008, Messias et al. 2012). Entretanto, representantes desta família foram coletados em regiões próximas à área de estudo: Vellozia mirabilis foi registrada em campos da Morraria do Amolar (acervo do Herbário COR) e Vellozia tubiflora em afloramentos graníticos na Bolívia (Porembski et al. 1998). Velloziaceae

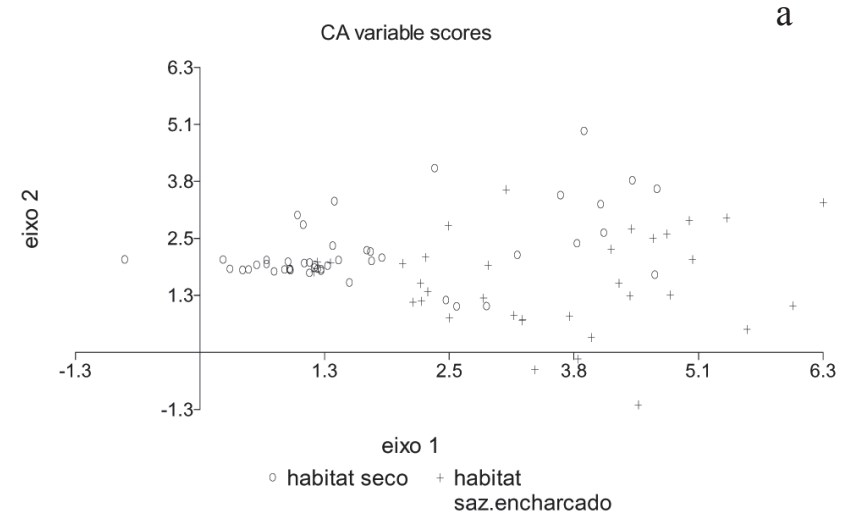

a possui centro de diversidade no sudeste brasileiro, especialmente nos afloramentos rochosos quartzíticos e areníticos, e o número de espécies desta família reduz-se drasticamente em direção às outras áreas. Carmo \& Jacobi (2013) também relataram a pouca influência de elementos florísticos de Eriocaulaceae e Xyridaceae em cangas do Estado de Minas Gerais (Viana \& Lombardi 2007, Jacobi \& Carmo 2008), de forma diversa do observado para os afloramentos quartzíticos da Cadeia do Espinhaço e Serra de São José (Carmo \& Jacobi 2013).

Sete espécies amostradas nas bancadas lateríticas de Corumbá perfizeram pouco mais de $75 \%$ dos valores de dominância, indicando que apenas $10 \%$ do total de espécies amostradas definem a estrutura desta comunidade, destacando-se a pteridófita Selaginella sellowii e a gramínea Sporobolus monandrus, as duas espécies com os maiores valores de importância.

Cerca de 30\% do total de espécies amostradas pertencem ao grupo de monocotiledôneas, como registrado em diversos afloramentos rochosos (Conceição et al. 2007, Scarano 2007). A presença marcante deste grupo de plantas em afloramentos rochosos denomina, inclusive, um tipo de habitat, as touceiras de monocotiledôneas ("monocotyledonous mats") (Porembski et al. 1997, Porembski 2007). Este padrão surge pela capacidade destas plantas sobreviverem em ambientes inóspitos e se reproduzirem

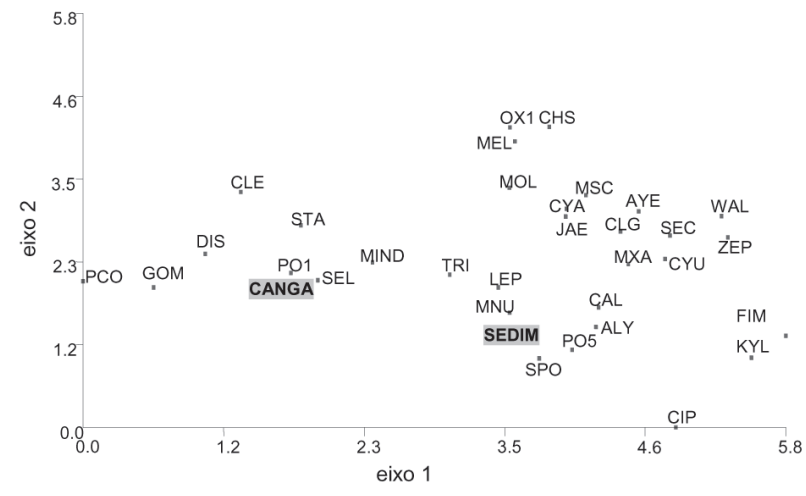

Figura 2. a. Interpolação dos escores dos dois primeiros eixos resultantes da DCA aplicada à matriz de valores de cobertura para 32 espécies amostradas em 81 parcelas nas bancadas lateríticas da fazenda Banda Alta, Ladário, MS, Brasil. Parcelas amostradas no "habitat seco" (o). Parcelas amostradas no "habitat sazonalmente inundado" (+). b. Resultados da DCA referentes às espécies e parcelas. CANGA: cobertura do substrato endurecido sem plantas; SEDIM: cobertura por sedimentos no interior da parcela. Os nomes abreviados das espécies constam na tabela 1 .

Figure 2. a. Ordination diagram of the plots and cover values of plant species obtained by detrended correspondence analysis (DCA) using data of 32 vascular plant species and 81 plots on ironstone outcrops of Banda Alta site, Ladário, Mato Grosso do Sul, Brazil. Plots of dry habitat (o). Plots of seasonally wet habitat (+). b. Results of detrended correspondence analysis ordination of plots and species. CANGA: hardened substrate without vascular plants; SEDIM: thin layer soil inside plot. Plant species are represented by abbreviation used in table 1. 
vegetativamente (Ibisch et al. 1995). Outro grupo bastante expressivo foram as pteridófitas, com cerca de $8 \%$ do total de espécies amostradas. Estas plantas, como, por exemplo Selaginella sellowii, podem ocorrer isoladamente ou em ilhas de solo sugerindo que mesmo espécies de plantas tolerantes à dessecação necessitem de certo grau de sombreamento, já que o incremento em biomassa deste tipo de planta é bastante lento.

Foram registradas apenas duas espécies endêmicas das bancadas lateríticas de Corumbá: Gomphrena centrota (Bortolotto et al. 1999, Pott et al. 2000) e Discocactus ferricola, uma cactácea elencada no rol de espécies ameaçadas da CITES, cuja distribuição geográfica é restrita à região de Corumbá e do Mutum, na Bolívia (Eggli 2002). Esta baixa frequência de espécies endêmicas também foi constatada para os afloramentos graníticos da Bolívia (Ibisch et al. 1995) e da Guiana Francesa (Sarthou \& Villiers 1998), bem como nas cangas da Serra de Carajás (Rayol 2006). Este resultado, entretanto, difere do que vem sendo registrado para os afloramentos rochosos do Sudeste brasileiro (Porembski et al. 1998), apontados por Scarano (2007) como um dos três hotspots de diversidade de plantas em afloramentos rochosos. Estudos recentes sobre a flora em cangas do Quadrilátero Ferrífero e do sudoeste da Austrália mostram elevada diversidade e endemismo (Jacobi et al. 2007, Gibson et al. 2010). O sudoeste da Austrália é citado como um hotspot de diversidade de espécies sobre afloramentos rochosos ferruginosos por Gibson et al. (2010), que atribuem este padrão de diversidade a fatores topográficos locais e o longo período em que esta paisagem não sofreu interferências de transgressões marinhas.

A baixa proporção de substrato ferruginoso endurecido, fragmentos de minério de ferro/manganês endurecidos ou sedimentos no interior das parcelas avaliadas $(25 \%)$ revelaram que a cobertura vegetal do estrato herbáceo nas bancadas lateríticas de Corumbá se dá de maneira relativamente contínua, resultado distinto ao constatado em outros estudos onde os afloramentos rochosos possuem maior proporção (p. ex. Caiafa \& Silva 2007, Neves \& Conceição 2007) e a ocorrência da vegetação restringe-se a depressões, fissuras ou fendas (p. ex. Ribeiro et al. 2007). Tal fato pode ser decorrente da posição topográfica das bancadas lateríticas de Corumbá nas regiões coluviais (altitudes entre 100 e $200 \mathrm{~m}$ ) e de sua baixa declividade, reduzindo a ação deletéria de enxurradas e contribuindo para o acúmulo de uma fina camada de sedimentos, especialmente nas depressões e cavidades em seu interior.

O estrato herbáceo estudado apresentou valores dos índices de diversidade de Shannon-Wienner e equabilidade próximos aos observados para outros

a
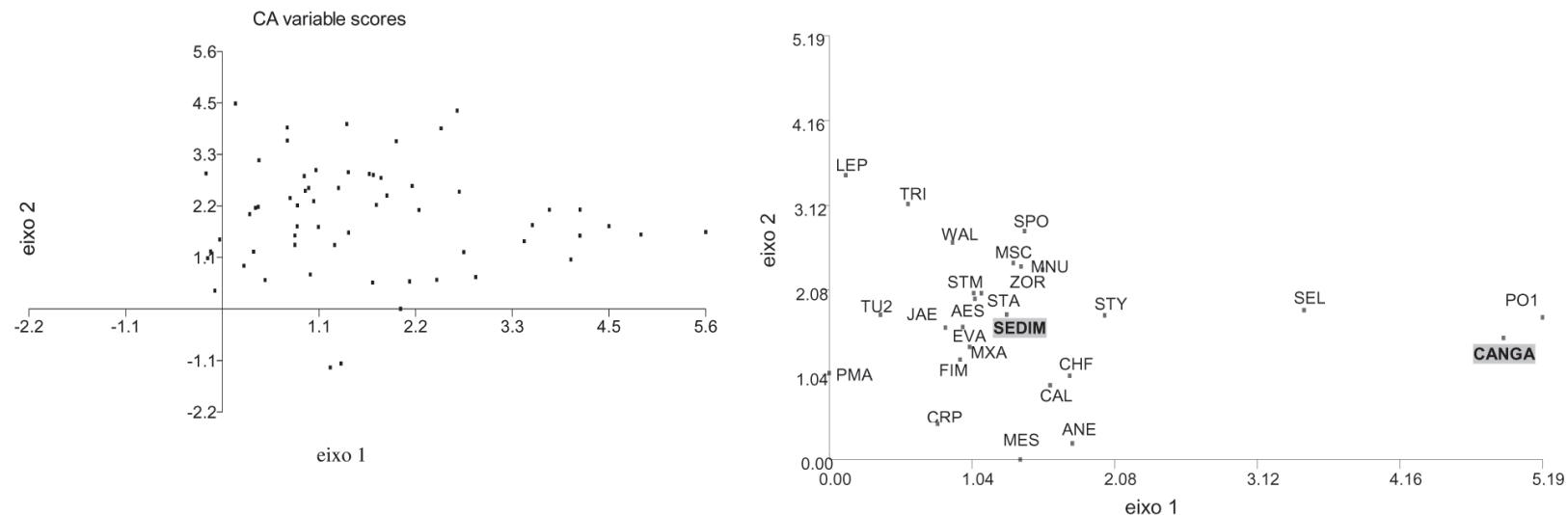

Figura 3. a. Interpolação dos escores dos dois primeiros eixos resultantes da DCA aplicada à matriz de valores de cobertura para 24 espécies amostradas em 60 parcelas nas bancadas lateríticas da fazenda São Sebastião do Carandá, Ladário, MS, Brasil. b. Resultados da DCA referentes às espécies e parcelas. CANGA: cobertura substrato endurecido sem plantas; SEDIM: cobertura por sedimentos no interior da parcela. Os nomes abreviados das espécies constam na tabela 1.

Figure 3. a. Ordination diagram of the plots and cover values of plant species obtained by detrended correspondence analysis (DCA) using data of 24 vascular plant species and 60 plots on ironstone outcrops of São Sebastião do Caranda site, Ladário, Mato Grosso do Sul, Brazil. Plant species are represented by abbreviation used in table 1. b. Results of detrended correspondence analysis ordination of plots and species. CANGA: hardened substrate without vascular plants; SEDIM: thin layer soil inside plot. Plant species are represented by abbreviation used in table 1 . 


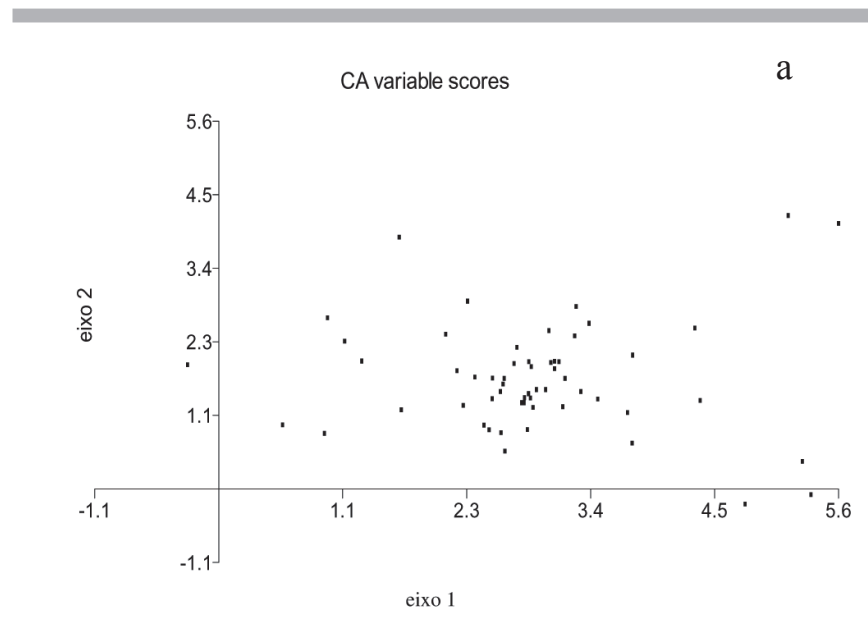

Figura 4. a. Interpolação dos escores dos dois primeiros eixos resultantes da DCA aplicada à matriz de valores de cobertura para 29 espécies amostradas em 58 parcelas nas bancadas lateríticas da fazenda Monjolinho, Corumbá, MS, Brasil. b. Resultados da DCA referentes às espécies e parcelas. CANGA: cobertura do substrato endurecido sem plantas; SEDIM: cobertura por sedimentos no interior da parcela; FRAG: fragmentos de minério de ferro endurecido. Os nomes abreviados das espécies constam na tabela 1.

Figure 4. a. Ordination diagram of the plots and cover values of plant species obtained by detrended correspondence analysis (DCA) using data of 29 vascular plant species and 58 plots on ironstone outcrops of Monjolinho, Corumbá, Mato Grosso do Sul, Brazil. Plant species are represented by abbreviation used in table 1. b. Results of detrended correspondence analysis ordination of plots and species. CANGA: hardened substrate without vascular plants; SEDIM: thin layer soil inside plot; FRAG: fragments of hardened iron ore. Plant species are represented by abbreviation used in table 1 .

afloramentos rochosos do Brasil (Conceição \& Pirani 2005, França et al. 2006), incluindo algumas comunidades vegetais sobre canga (Jacobi et al. 2008, Messias et al. 2012).

Os arranjos revelados nas análises de ordenação sugerem um gradiente ambiental nas bancadas lateríticas de Corumbá, onde algumas espécies se estabelecem em locais com maior proporção de canga exposta e outras sobre uma fina camada de substrato, como já apontado por Vincent \& Meguro (2008); as primeiras suportam condições de alta insolação, temperaturas elevadas e escassez de água, típicas dos afloramentos rochosos; as espécies que ocorrem diretamente sobre o substrato endurecido também podem se estabelecer em ilhas de solo onde, muitas vezes, ocupam suas bordas, mas o inverso não ocorre.

Pequenas quantidades de sedimentos acumulados em depressões, cavidades, fissuras ou fendas nas cangas propiciam o surgimento de espécies mais exigentes. Isto é particularmente observado na canga nodular da fazenda São Sebastião do Carandá, onde as espécies Portulaca mucronulata e Selaginella sellowii limitam-se aos pontos com substrato ferruginoso endurecido aflorante. $\mathrm{O}$ habitat "sazonalmente inundado" da fazenda Banda Alta encontra-se em uma posição topográfica que propicia o depósito coluvional, bem como recebe grande parte do fluxo de águas pluviais durante a estação chuvosa. Tais condições permitem o estabelecimento de espécies efêmeras ou geófitas típicas de ambientes sazonalmente inundados e/ ou que se estabelecem sobre um substrato mais profundo, como Cipura formosa, Fimbristylis sp., Ludwigia spp. e Zephyranthes cearensis. Estudos em campos rupestres ferruginosos do Quadrilátero Ferrífero também constataram que propriedades físicas, como a ocorrência de rochas expostas e características granulométricas, podem ser determinantes na composição de espécies das comunidades (Messias et al. 2013).

Estes resultados sugerem que a vegetação herbácea das bancadas lateríticas de Corumbá se distribui ao longo de um gradiente ambiental determinado pela granulometria do substrato, com espécies que se estabelecem diretamente sobre substrato ferruginoso endurecido e outras que colonizam locais com acúmulo de sedimentos. Deve-se levar em conta a existência da heterogeneidade ambiental destas cangas, em uma escala mais fina, evitando-se considerar esta vegetação como uma comunidade homogênea espacial e temporalmente. Destaca-se, ainda, a importância da conservação da flora das bancadas lateríticas de Corumbá, tanto pelo seu caráter insular quanto pela escassez de informações sobre estes ecossistemas que constituem o mosaico de paisagens associados ao Pantanal. 


\section{Agradecimentos}

À CAPES, pela Bolsa de Doutoramento pelo Programa de Pós-Graduação em Ecologia da Universidade de São Paulo concedida à primeira Autora; aos proprietários das fazendas, Srs. Altair Gonçalo, Luis Alberto Pinto de Figueiredo e Sami Loft, por permitirem o acesso às áreas de estudos; aos pesquisadores que identificaram o material botânico, Adriana Guglieri (Poaceae-UFMS), Ana Maria Goulart de A. Tozzi (Fabaceae-UNICAMP), Alexa Oliveira Paes Coelho (Portulacaceae-UNEB), Claudia E. Carneiro (Caryophyllaceae-UEFS), Elton M. Assis (PteridophytaUFMS), Gerleni Lopes (Malvaceae-IBt), Jumara Marques Souza (Oxalidaceae-UEFS), Juliana Lovo (IridaceaeUSP), Marcela Firens (Rubiaceae-UNICAMP), Marcos José da Silva (Euphorbiaceae, PhyllanthaceaeUNICAMP), Maria Candida Mamede (MalpighiaceaeIBt), Maria do Carmo Amaral (CommelinaceaeUNICAMP), Rafael Trevisan (Cyperaceae-UFRGS), Renata Oliveira (Amaryllidaceae-UNICAMP), Renata Sebastiani (Malpighiaceae-Janusia-IBt), Rosangela S. Bianchini (Convolvulaceae-IBt), Rosilene Silva (Fabaceae-UNICAMP), Rubens T. Queiroz (FabaceaeUNICAMP).

\section{Literatura citada}

APG III. 2009. An update of the Angiosperm Phylogeny Group classification for the orders and families of flowering plants: APG III. Botanical Journal of the Linnean Society 161: 105-121.

Augustin, C.H.R.R., Lopes, M.R.S. \& Silva, S.M. 2013. Lateritas: um conceito ainda em construção. Revista Brasileira de Geomorfologia 14: 241-257.

Baker, A.J.M. \& Brooks, R.R. 1988. Botanical exploration for minerals in the humid tropics. Journal of Biogeography 15: 221-229.

Barthlott, W., Groger, A. \& Porembski, S. 1993a. Some remarks on the vegetation of tropical inselbergs: diversity and ecological differentiation. Biogéographica 69:17-36.

Barthlott, W., Porembski, S., Szarzynski, J. \& Mund, J.P. 1993b. Phytogeography and vegetation of tropical inselbergs. Actes du colloque international de Phytogéographie tropicale, pp.15-24.

Bigarella, J.J., Becker, R.D. \& Santos, G.F. 2007. Estrutura e origem das paisagens tropicais e subtropicais. 2 ed., v.1. Editora da UFSC, Florianópolis.

Bortolotto, I.M., Damasceno-Júnior, G.A. \& Isquierdo, S.W.G. 1999. Caracterização das unidades fitofisionômicas da bacia da Lagoa Negra em Ladário MS. In: Anais do $2^{\circ}$ Simpósio sobre Recursos Naturais e Socioeconômicos do Pantanal: manejo e conservação, Embrapa, Brasília, pp. 283-289.
Brummitt, R.K. \& Powell, C.E. 1992. Authors of plant names. Royal Botanical Gardens, Kew.

Burbanck, M.P. \& Platt, R.B. 1964. Granite outcrop communities of the piedmont plateau in Georgia. Ecology 45: 292-306.

Caiafa, A.N. \& Silva, A.F. 2007. Structural analysis of the vegetation on a highland granitic rock outcrop in Southeast Brazil. Revista Brasileira de Botânica 30: 657-664.

Cardoso, E.L., Oliveira, H., Amaral, J.A.M., Ker, J.C., Pereira, N.R., Santos, R.D., Tôsto, S.G., Spera, S.T. \& Carvalho Júnior, W. 2000. Pedologia. In: J.S.V. Silva (org.). Zoneamento ambiental da borda oeste do Pantanal: maciço do Urucum e adjacências. Embrapa Comunicação para Transferência de Tecnologia, Brasília, pp. 95-109.

Carmo, F.F. \& Jacobi, C.M. 2013. A vegetação de canga no Quadrilátero Ferrífero, Minas Gerais: caracterização e contexto fitogeográfico. Rodriguésia 64: 527-541.

Carmo, F.F., Campo, I.C. \& Jacobi, C.M. 2012. Cangas: ilhas de ferro estratégicas para a conservação. Ciência Hoje 295: 48-53.

Cleef, A. \& Silva, M.F.F. 1994. Plant communities of the Serra dos Carajás (Pará), Brazil. Boletim do Museu Paraense Emílio Goeldi, série Botânica, 10: 269-281.

Conceição, A.A. \& Pirani, J.R. 2005. Delimitação de habitats em campos rupestres na Chapada Diamantina, Bahia: substratos, composição florística e aspectos estruturais. Boletim de Botânica da Universidade de São Paulo 23: 85-111.

Conceição, A.A., Pirani, J.R. \& Meirelles, S.T. 2007. Floristics, structure and soil of insular vegetation in four quartzite-sandstone outcrops of "Chapada Diamantina", northeast Brazil. Revista Brasileira de Botânica 30: 641-656.

Eggli, U. 2002. Synopsis of the Cactaceae of Mato Grosso, Brazil. Haseltonia 9: 146-166.

França, F., Melo, E. \& Gonçalves, J.M. 2006. Aspectos da diversidade da vegetação no topo de um inselbergue no semi-árido da Bahia, Brasil. Sitientibus, série Ciências Biológicas 6: 30-35.

Gibson, N., Yates, C.J. \& Dillon, R. 2010. Plant communities of the ironstone ranges of South Western Australia: hotspots for plant diversity and mineral deposits. Biodiversity Conservation 19: 3951-3962.

Ginocchio, R. \& Baker, A.J.M. 2004. Metallophytes in Latin America: a remarkable biological and genetic resource scarcely known and studied in the region. Revista Chilena de Historia Natural 77: 185-194.

Hambler, D.J. 1964. The vegetation of granitic outcrops in western Nigeria. Journal of Ecology 52: 573-594.

Ibisch, P.L., Rauer, G., Rudolph, D. \& Barthlott, W. 1995. Floristic, biogeographical, and vegetational aspects of Pre-Cambrian rock outcrops (inselbergs) in eastern Bolivia. Flora 190: 299-314. 
Jacobi, C.M. \& Carmo, F.F. 2008. The contribution of ironstone outcrops to plant diversity in the Iron Quadrangle, a threatened brazilian landscape. Ambio 37: 324-326.

Jacobi, C.M., Carmo, F.F. \& Vincent, R.C. 2008. Estudo fitossociológico de uma comunidade vegetal sobre canga como subsídio para a reabilitação de áreas mineradas no Quadrilátero Ferrífero, MG. Revista Árvore 32: 345-353.

Jacobi, C.M., Carmo, F.F., Vincent, R.C. \& Stehmann, J.R. 2007. Plant communities on ironstone outcrops: a diverse and endangered Brazilian ecosystem. Biodiversity and Conservation 16: 2185-2200.

Krebs, C.J. 1972. Ecology: the experimental analysis of distribution and abundance. Harper and Row, New York.

Legendre, P. \& Legendre, L. 1998. Numerical ecology. 2 ed. Elsevier, Amsterdan.

Lista de espécies da Flora do Brasil. 2014. Jardim Botânico do Rio de Janeiro. Disponível em <http:// floradobrasil.jbrj.gov.br/> (acesso em 20-VII-2012).

Meirelles, S.T., Pivello, V.R. \& Joly, C.A. 1999. The vegetation of granite rock outcrops in Rio de Janeiro, Brazil, and the need for its protection. Environmental Conservation 26: 10-20.

Messias, M.C.T.B., Leite, M.G.P., Meira-Neto, J.A.A. \& Kozovits, A.R. 2012. Fitossociologia de campos rupestres quartzíticos e ferruginosos no Quadrilátero Ferrífero, Minas Gerais. Acta Botanica Brasilica 26: 230-242.

Messias, M.C.T.B., Leite, M.G.P., Meira Neto, J.A.A., Kozovits, A.R. \& Tavares, R. 2013. Soil-vegetation relationship in quartzitic and ferruginous Brazilian rocky outcrops. Folia Geobotanica 48: 509-521.

Mourão, A. \& Stehmann, J.R. 2007. Levantamento da flora do campo rupestre sobre canga hematítica couraçada remanescente na Mina do Brucutu, Barão de Cocais, Minas Gerais, Brasil. Rodriguésia 58: 775-786.

Mueller-Dombois, D. \& Ellenberg, H. 1974. Aims and methods of vegetation ecology. Willey, New York.

Neves, S.P.S. \& Conceição, A.A. 2007. Vegetação em afloramentos rochosos na Serra do Sincorá, Chapada Diamantina, Bahia, Brasil. Sitientibus série Ciências Biológicas 7: 36-45.

Phillips, D.L. 1981. Succession in granite outcrop shrubtree communities. The American Midland Naturalist 106: 313-317.

Porembski, S. 2007. Tropical inselbergs: habitat types, adaptative strategies and diversity patterns. Revista Brasileira de Botânica 30: 579-586.

Porembski, S., Barthlott, W., Dörrstock, S. \& Biedinger, N. 1994. Vegetation of rock outcrops in Guinea: granite inselbergs, sandstone table mountains and ferricretes - remarks on species number and endemism. Flora 189: 315-326.
Porembski, S., Martinelli, G., Ohlemüller, R. \& Barthlott, W. 1998. Diversity and ecology of saxicolous vegetation mats on inselbergs in the Brazilian Atlantic rainforest. Diversity and Distributions 4: 107-119.

Porembski, S., Seine, R. \& Barthlott, W. 1997. Inselberg vegetation and the biodiversity of granite outcrops. Journal of the Royal Society of Western Australia 80: 193-199.

Porto, M.L. \& Silva, M.F.F. 1989. Tipos de vegetação metalófila em áreas da Serra de Carajás e de Minas Gerais, Brasil. Acta Botanica Brasilica 3: 13-21.

Pott, A., Silva, J.S.V., Salis, S.M., Pott, V.J. \& Silva, M.P. 2000. Vegetação e uso da terra. In: J.S.V. Silva (org.). Zoneamento ambiental da borda oeste do Pantanal: maciço do Urucum e adjacências. Embrapa Comunicação para Transferência de Tecnologia, pp. 111-131.

Rayol, B.P. 2006. Análise florística e estrutural da vegetação xerofítica das savanas metalófilas na Floresta Nacional de Carajás: subsídios à conservação. Dissertação de Mestrado, Universidade Rural da Amazônia e Museu Paraense Emílio Goeldi, Pará.

Ribeiro, K.T., Medina, B.M.O. \& Scarano, F.R. 2007. Species composition and biogeographic relations of the rock outcrop flora on the high plateau of Itatiaia, SE-Brazil. Revista Brasileira de Botânica 30: 623-639.

Rizzini, C.T. 1997. Tratado de fitogeografia do Brasil: aspectos ecológicos, sociológicos e florísticos. 2. ed. Âmbito Cultural Edições Ltda., Rio de Janeiro.

Sarthou, C.\& Villiers, J.F. 1998. Epilithic plant communities on inselbergs in French Guiana. Journal of Vegetation Science 9: 847-860.

Sarthou, C., Kounda-Kiki, C., Vaçulik, A., Mora, P. \& Ponge, J.F. 2009. Successional patterns on tropical inselbergs: A case study on the Nouragues inselberg (French Guiana). Flora 204: 396-407.

Scarano, F.R. 2007. Rock outcrop vegetation in Brazil: a brief overview. Revista Brasileira de Botânica 30: 561-568.

Secco, R.S. \& Lobo, M.G.A. 1988. Considerações taxonômicas e ecológicas sobre a flora dos "campos rupestres" da Serra dos Carajás (PA). Boletim da FBCN 23: 30-44.

Secco, R.S. \& Mesquita, A.L. 1983. Notas sobre a vegetação de canga na Serra Norte - I. Boletim do Museu Paraense Emílio Goeldi 59: 1-13.

Seine, R., Becker, U., Porembski, S., Follmann, G. \& Barthlott, W. 1998. Vegetation of inselbergs in Zimbabwe. Edinburgh Journal of Botany 55: 267-293.

Shure, D. \& Ragsdale, H.L. 1977. Patterns of primary succession on granite outcrop surfaces. Ecology 58: 993-1006. 
Silva, M.F.F. 1991. Análise florística da vegetação que cresce sobre canga hematítica em Carajás - Pará (Brasil). Boletim do Museu Paraense Emílio Goeldi, série Botânica 7: 79-107.

Silva, M.F.F. 1992. Distribuição de metais pesados na vegetação metalófita de Carajás. Acta Botanica Brasilica 6: 107-122.

Silva, M.F.F., Secco, R.S. \& Lobo, M.G. 1996. Aspectos ecológicos da vegetação rupestre da Serra dos Carajás, Estado do Pará, Brasil. Acta Amazonica 26: 17-44.

Silva, R.R. \& Tozzi, A.M.G.A. 2008. A new species of Lonchocarpus (Leguminosae, Papilionoideae) from Mato Grosso do Sul, Brazil. Brittonia 60: 34-37.

Soriano, B.M.A. 2000. Climatologia. In: J.S.V. Silva (org.). Zoneamento ambiental da borda oeste do Pantanal: maciço do Urucum e adjacências. Embrapa Comunicação para Transferência de Tecnologia, pp. 69-81.
Takahasi, A. 2010. Ecologia da vegetação em bancadas lateríticas em Corumbá, MS. Tese de doutorado, Universidade de São Paulo, São Paulo.

The plant list. 2014. Version 1.1. Disponível em $<$ http:// www.theplantlist.org/> (acesso em 10-V-2012).

Tropicos.org. 2014. Missouri Botanical Garden. Disponível em <http://tropicos.org> (acesso em 23-III-2013).

Viana, P.L. \& Lombardi, J.A. 2007. Florística e caracterização dos campos rupestres sobre canga na Serra da Calçada, Minas Gerais, Brasil. Rodriguesia 58: 159-177.

Vincent, R.C. \& Meguro, M. 2008. Influence of soil properties on the abundance of plant species in ferruginous rocky soils vegetation, southeastern Brazil. Revista Brasileira de Botânica 31: 377-388. 